present study finds that the likelihood of seizure recurrence is $10 \%$ in ADHD patients with treated epilepsy who receive MPH. In ADHD patients with abnormal EEG without clinical seizures, MPH did not appear to cause seizures and may have a normalizing effect on the EEG (see also Swartwood MO et al. Pediatr Neurol 1998;18:244-250). The prevalence of abnormal EEG in patients with ADHD is reviewed in Ped Neur Briefs Oct 2000;14:73-74.

Hemmer SA, Pasternak JK, et al found that $15.4 \%$ of nonepileptic children with ADHD demonstrated epileptiform abnormalities in the EEG (Pediatr Neurol 2001;24:99102). During treatment with MPH, seizures occurred in 1 of 175 nonepileptic ADHD patients with normal EEG (0.6\%), and in 3 of 30 with epileptiform EEGs $(10 \%)$. Of 12 children with rolandic spikes, $2(16.7 \%)$ developed seizures with MPH. A normal EEG indicates minimal risk of seizures with MPH therapy for ADHD. In contrast, an epileptiform EEG in nonepileptiform children with ADHD predicts a $10-17 \%$ risk of occurrence of seizures. The prophylactic value of AED treatment in these ADHD patients must be weighed against the potential adverse effects of AED, especially on cognition. My own present policy is to withhold AED "umbrella" therapy with MPH. Atomoxetine (Strattera), the new nonstimulant treatment for ADHD, may prove advantageous in these cases, but its effect on the EEG remains to be established.

\title{
CLASSIFICATION, DIAGNOSIS, AND TREATMENT OF SYNCOPE
}

The classification, diagnosis, investigation, and management of syncopal attacks in childhood are reviewed from the Department of Cardiology, Royal Hospital for Sick Children, Glasgow, UK. The causes of childhood syncope are classified in three groups: neurally mediated, cardiovascular, and noncardiovascular pseudo-syncope. Neurallymediated (neurocardiogenic) syncopes are most commonly reflex in type, and consist of a transient disturbance in the autonomic control of heart rate and blood pressure. They occur at any age, typically in toddlers and in children between 9 and 14 years of age. Characteristically, the child is either sitting or standing, and suffers loss of tone and consciousness, preceded by dizziness, nausea, and pallor. Hypotension and/or bradycardia result in cerebral hypoxia, and when severe, an anoxic seizure and incontinence. The reflex anoxic seizure resembles a pallid breath-holding attack in a toddler, and consists of opisthotonus and twitching. Unlike an epileptiform discharge, the electroencephalographic (EEG) record is flat during a reflex anoxic seizure.

The diagnosis may be made by a detailed and careful history, and investigations are usually normal. For recurrent syncope, a 12 lead electrocardiogram is advised, primarily to exclude a long QT interval. "Warning bells" that prompt investigation include syncope in response to fright or exercise, syncope while supine, and a family history of sudden unexplained death. Tests such as an echocardiogram or EEG are usually not indicated in a single or uncomplicated attack; they should be considered principally in patients with other cardiac or neurologic symptoms and signs. A tilt test is positive in approximately $50 \%$ of children age 6 years or older with recurrent neurally-mediated syncope, and cardiac event monitoring can be a productive investigation in the toddler age group with reflex asystolic syncope. In psychogenic pseudosyncope, the ECG, EEG, and blood pressure are normal during continuous ambulatory inpatient monitoring. Management of neurally-mediated syncope consists of reassurance, plenty of fluids and added salt, and maintenance of blood pressure by crossing legs and folding the arms. Drug therapy is considered when simple 
measures fail. Fludrocortisone is the initial choice, and a beta blocker is added if attacks are unresponsive. Neither drug has been tested by randomized clinical trial. Iron therapy may be helpful in toddlers with neurocardiogenic syncope associated with iron deficiency. Atropine reduces the severity and frequency of syncope, but side effects are common. For older children with frequent syncope unresponsive to drug therapy, a cardiac pacemaker may prevent an associated bradycardia or asystole. (McLeod KA. Syncope in childhood. Arch Dis Child 2003;88:350-353). (Respond: Dr KA McLeod, Consultant in Paediatric Cardiology, Department of Cardiology, Royal Hospital for Sick Children, Yorkhill NHS Trust, Glasgow G3 85J, UK).

COMMENT. The cardiologist's classification of syncope differs from that of the neurologist, who usually reserves the term simple neurocardiogenic syncope for episodes of fainting that occur in older children and are precipitated by pain, fear, excitement, and extended periods of standing, particularly in a warm environment. (Haslem RHA. Conditions that mimic seizures. In Nelson Textbook of Pediatrics. 16 ${ }^{\text {th }}$ ed, Philadelphia, WB Saunders, 2000;p1830). Simple syncope results from vasovagal stimulation and is characterized by a prodrome of nausea, pallor, diaphoresis, and blurred vision. It is uncommon before 10-12 years of age, and is prevalent in adolescent females. Simple syncope is differentiated from an epileptiform seizure by the complete orientation soon after the event, and the absence of seizure discharges in the EEG during an attack, but the distinction is sometimes difficult. In toddlers, syncope is described under breathholding spells, especially the pallid variety. Breath-holding differs from simple syncope in the age of onset, precipitation by a startle or painful injury, and the sudden interruption of breathing that precedes loss of consciousness. Cough syncope results from an increase in intrapleural pressure, most common in asthmatic children. A coughing spell usually during sleep is accompanied by loss of consciousness, hypotonia, vertical eye-rolling, and clonic convulsive movements of short duration.

Role of the Tilt Test. In patients with a history consistent with neurocardiogenic (vasovagal) syncope, an extensive workup that currently often includes EEG, ECG, MRI, echocardiogram, etc, is not routinely indicated. In those with recurrent syncope, the tilt table testing performed early will increase the frequency of a definitive diagnosis, and avoids the inconvenience and expense of further investigations. In a retrospective analysis of 54 consecutive patients with recurrent syncope, evaluated with or without tilt table testing, 27 patients without tilt table tests received a greater number of neurology consultations, EEGs, and CTs, but a positive diagnosis was made in only $5(18 \%)$. In contrast, a diagnosis was made early in all of 27 patients tested by tilt table, 25 having neurocardiogenic syncope and 2 conversion reactions (Strieper MJ et al. Pediatrics 1994;93:660-662; see Progress in Pediatric Neurology III, PNB Publishers, 1997;pp464465).

\section{MOVEMENT DISORDERS}

\section{PATHOGENESIS OF OPSOCLONUS MYOCLONUS}

The proceedings of the $2^{\text {nd }}$ International Opsoclonus Myoclonus Ataxia Syndrome (OMAS) Symposium (Feb 6-8, 2003; Abingdon, Oxfordshire, UK) are summarized from 